\title{
Investigation of Predictive Factors for Patients with Nondiagnostic or Insufficient FNAB Results Who Underwent Thyroidectomy
}

Ibrahim KILINC', Mustafa OZSOY2, Fahri YETISIR ${ }^{3}$

${ }^{1}$ Department of General Surgery, Ankara City Hospital, Bilkent, Ankara, Turkey.

2 Department of General Surgery, Faculty of Medicine, Yıldırım Beyazıt University, Altindag, Ankara, Turkey.

${ }^{3}$ Department Of General Surgery, V-M Medicalpark Hospital, Ankara, Turkey

Correspondence

Ibrahim KILINC

Department of General Surgery, Ankara City Hospital, Bilkent, Ankara, Turkey.

e-mail:ikilinc8083@gmail.com

\section{ABSTRACT}

Thyroid fine-needle aspiration biopsy (FNAB) is used in managing patients with thyroid nodules. Therefore, this study aimed to investigate the predictive factors for malignancy.

The study included 171 patients whose FNAB results were interpreted as nondiagnostic twice or more and who had previously undergone a surgical treatment. Patients were evaluated in terms of demographic characteristics; serum thyroglobulin, antithyroglobulin, and anti-thyroid peroxidase levels, and ultrasound features.

A statistically significant difference was found between malign and benign patients in terms of anti-thyroglobulin levels and hypoechogenicity. However, no significant difference was observed in terms of age; sex; anti-thyroidperoxidase and thyroglobulin levels; nodule diameter; solid, cystic, or mixed features; and other ultrasonographic features.

Hypoechogenity and anti-thyroglobulin levels can be used as predictive factors in terms of malignancy for patients whose FNAB results were nondiagnostic two or more times.

Key words: Anti-thyroglobulin, malignancy, nondiagnostic, thyroid fine-needle aspiration biopsy.

\section{INTRODUCTION}

Thyroid fine-needle aspiration biopsy (FNAB) is the most valuable, accurate, and inexpensive method for patients with thyroid nodules (1-3). Most biopsies are adequate for cytological diagnosis. However, $2 \%-$ $18 \%$ of these biopsies are reported as nondiagnostic, and of these nondiagnostic biopsies, $1 \%-4 \%$ include the risk of malignancy (4). The American Thyroid Association and Bethesda System for Reporting Thyroid Cytopathology suggest performing an ultrasoundguided repeat FNAB. However, the second FNAB may also yield an inadequate result. Thus, the management of these cases is essentially determined according to the malignancy risks they include $(5,6)$. The aim of this study was to investigate the risk of malignancy in patients whose FNAB results were interpreted as nondiagnostic two times or more and who had undergone a surgical treatment.

\section{MATERIAL AND METHODS}

Data obtained from digital records and patient files of 171 patients who had previously undergone a surgical treatment were included in this retrospective study. Thyroid nodules from these patients were initially evaluated using an Esaote color Dopler ultrasound (USG) (model: 796FDIl; MAG Technology Co, Ltd, Yung-ho City, Taipei, Taiwan) and a superficial probe (model no: LA523 13-4, 5.5-12.5 Mhz). For repeat FNAB, a General Electric Logiq Pro 200 (model no: 2270968; GE Healthcare Korea, Seongnam-si, Gyeonggi-do, Korea) and a 5.5- to 7.5-MHz probe were used. Biopsy was performed using a $25 \mathrm{G}$ needle attached to a $10-\mathrm{mL}$ syringe. All statistical analyses in this study were performed using SPSS (Windows 15,0) statistical package program. A chi-square test was used for categorical variables. $P$ values were compared with 0.05 value and examined with the $95 \%$ confidence level. Independent samples were analyzed using the $t$ test. 


\section{RESULTS}

Of the 171 patients included in the study, 45 (26.3\%) were malignant whereas 126 (73.7\%) were benign. Further, 125 patients (73.1\%) were female and $46(26.9 \%)$ were male. The average age of the patients was 50.63 years. No significant difference was found between malignant and benign results in terms of age and sex (Table 1). When the preoperative thyroglobulin, anti-thyroglobulin (anti-TG), and anti-thyroid peroxidase levels were assessed, significant differences were observed between the groups in terms of anti-thyroglobulin level (Table 2). No difference was noted between the groups in terms of nodule diameter (Table 3). When ultrasonographic characteristics of the nodule were analyzed, no difference was found between malignant and benign groups in terms of solid, cystic, mixed, isoechogenicity, hyperechogenicity, halo formation, irregular margin, and microcalcification features of the nodule. However, a significant cor- relation was observed between malignancy and hypoechogenicity characteristics of the nodule (Tables 4 and 5).

\section{DISCUSSION}

In a thyroid nodule, malignancy is more probable in the presence of ultrasonographic features such as hypoechogenity, increased vascularization, marginal irregularity, presence of microcalcification, absence of halo, and nodule height being greater than the transverse dimension $(7,8)$. FNAB plays a critical role in assessing these patients and has reduced the rate of unnecessary surgical procedures (9). It is superior in managing thyroid nodules compared with other diagnostic instruments due to its 93\% sensitivity, 95.1\% specificity, $88.9 \%$ positive predictive value, and $96.5 \%$ negative predictive value (10). Cases shown to be benign through FNBA are generally managed us-

Table 1 Analysis of age and sex results

\begin{tabular}{lllll}
\hline & Total cases & Benign & Malign & $P$ value \\
\hline Gender & Female: 125 & $93(74.4)$ & $32(25.06)$ & 0.726 \\
Male: 46 & $33(71.7)$ & $13(28.3)$ & \\
Age & 171 & Average: 50.10 & Average: 51.16 & 0.637 \\
\hline
\end{tabular}

Table 2 Assessment in terms of thyroglobulin (TG), anti-thyroglobulin (anti-TG), and antithyroid peroxidase level (anti-TPO)

\begin{tabular}{lllll}
\hline Parameter & & Total cases & Average & $P$ value \\
\hline TG & Benign & 95 & $78.44 \mathrm{ng} / \mathrm{ml}$ & 0.634 \\
& Malign & 25 & $77.36 \mathrm{ng} / \mathrm{ml}$ & 0.022 \\
\hline \multirow{2}{*}{ Anti-TG } & Benign & 120 & $78.38 \mathrm{IU} / \mathrm{ml}$ & $27.37 \mathrm{IU} / \mathrm{ml}$ \\
& Malign & 41 & $47.71 \mathrm{IU} / \mathrm{ml}$ & 0.282 \\
\hline
\end{tabular}

Table 3 Description of nodule diameter

\begin{tabular}{l|l|l|l}
\hline & Total cases & Mean $(\mathrm{mm})$ & $P$ value \\
& Benign: 126 & 20.76 & 0.557 \\
& Malign: 45 & 17.36 & \\
\hline
\end{tabular}


Table 4 Radiological examination of the nodule with echogenicity, halo formation, border irregularity, and microcalcification

\begin{tabular}{|c|c|c|c|c|}
\hline & Total cases & Benign & Malign & $P$ Value \\
\hline & Absent: 106 & 77 (72.7\%) & $29(27.3 \%)$ & \\
\hline \multirow[t]{2}{*}{ Isohypoechogenicity } & Presence: 65 & 49 (75.3\%) & $16(24.7 \%)$ & 0.635 \\
\hline & Absent:83 & 57 (68.6\%) & $26(31.4 \%)$ & \\
\hline \multirow[t]{2}{*}{ Isoechogenicity } & Presence: 88 & 69 (78.4\%) & $19(21.6 \%)$ & 0.129 \\
\hline & Absent: 153 & $120(78.5 \%)$ & 33 (21.5\%) & \\
\hline \multirow[t]{2}{*}{ Hypoechogenıcity } & Presence: 18 & $6(23.4 \%)$ & $12(66.6 \%)$ & 0.000 \\
\hline & Absent:170 & 125 (73.5\%) & 45 (26.5\%) & \\
\hline \multirow[t]{2}{*}{ Hyperechogenıcıty } & Presence: 1 & $1(100 \%)$ & 0 & - \\
\hline & Absent: 118 & $92(78 \%)$ & $26(22 \%)$ & \\
\hline \multirow[t]{2}{*}{ Halo Formatıon } & Presence: 53 & $34(64.1 \%)$ & 19 (35.9\%) & 0.058 \\
\hline & Absent: 81 & $56(69.1 \%)$ & 25 (30.9\%) & \\
\hline \multirow[t]{2}{*}{ Border Irregularity } & Presence: 90 & 70 (77.7\%) & $20(22.3 \%)$ & 0.200 \\
\hline & Absent: 115 & $80(69.6 \%)$ & 35 (30.4\%) & \\
\hline Halo Formation & Presence: 56 & $46(82.1 \%)$ & 10 (17.9\%) & 0.080 \\
\hline
\end{tabular}

Table 5 Description of ultrasonographic features of nodules

\begin{tabular}{lllll}
\hline & Total cases & Benign & Malign & P value \\
\hline Solid & 62 & $44(70.9 \%)$ & $18(29.1 \%)$ & 0.660 \\
Cystic & 6 & $5(83.3 \%)$ & $1(16.7 \%)$ & 0.585 \\
Mix & 103 & $77(74.7 \%)$ & $26(25.3 \%)$ & 0.283 \\
\hline
\end{tabular}

ing medical methods, while those determined to be neoplastic or malignant are treated surgically (11). However, currently, no consensus exists regarding the management of cases reported to be nondiagnostic due to the lack of adequate cellular component and of the cases reported as indeterminate for neoplasm due to overlapping cytological features between benign and neoplastic thyroid lesions $(5,12)$. According to the algorithm of the American Thyroid Association (ATA) regarding thyroid nodules, repeat FNA accompanied by ultrasound is strongly recommended for cases initially interpreted to be nondiagnostic, resulting in higher ratios of diagnoses. Although these rates are $75 \%$ in solid nodules and $50 \%$ in cystic nodules (13), still a small number of patients (7\%) are interpreted as nondiagnostic after repeat FNAB, with their nodules being determined as malignant using surgical procedures (14). A third attempt at FNAB in these cases is less likely to be diagnostic. FNAB reported as nondiagnostic for the second time is the point at which clinicians are faced with a dilemma (13). Clinicians must decide, as indicated by the ATA criteria, whether to continue with close observation or surgical resection for partially cystic nodules, or, if the nodule presents as sold, opt for surgery.

Moon et al. attempted to determine the parameters indicating malignancy by using data from 104 patients whose second FNA results were reported to be nondiagnostic (15). They found the malignancy rate for solid and cystic nodules to be $13 \%$ and $8.6 \%$ respectively. They also found that the irregular margin feature occurred more frequently in malignant 
nodules, while no significant difference was found in terms of hypoechogenicity and microcalcification between malign and benign nodules. Based on these results, the authors asserted that it is diagnostically more preferable to perform a combined assessment, rather than evaluating suspicious ultrasound findings per se. They also determined that cases with one or more suspicious findings (irregular margin, hypoechogenicity, and microcalcification) compared with cases without any suspicious findings had a malignancy risk of $25.7 \%$ and $4.3 \%$, respectively. Therefore, they stated that surgical treatment should be considered for cases with suspicious ultrasonography findings and defined as nondiagnostic for the second time. This was corroborated by Rosario et al. who evaluated 158 patients whose second FNAB results were nondiagnostic. They stated that the parameter of suspicious ultrasound findings could be defined as a predictive factor in terms of malignancy in patients with a nondiagnostic repeat FNA result and that patients reported to have this factor should be surgically treated (16).

In the present study, a significant inverse relationship was found between anti-thyroglobulin levels and malignancy. This level was determined to be lower in malign patients. Thus, anti-thyroglobulin levels can guide the treatment of these patients. This result is particularly important for patients with thyroiditis because these patients have high anti-thyroglobulin levels, and it is difficult to distinguish whether FNAB of these patients is malign or benign. In this study, malign and benign patients were compared in terms of the diameter of nodules, and no difference between these two groups was determined. Patients were also separately evaluated based on whether their nodule structures were solid, cystic, or mixed. Once again, no significant difference was found in these three parameters and malignancy. Isoechogenicity, isohypoechogenicity, hypoechogenicity, and hyperechogenicity were evaluated as patterns of echogenicity. Among these, a significant relationship was determined between hypoechogenicity and malignancy. Accordingly, hypoechogenicity may be a surgical criterion per se in patients whose second FNAB results are nondiagnostic. However, the present study had some restrictions. Patients whose FNAB results were reported as nondiagnostic at least two times and only those who had undergone surgical treatment were included in this study. Also, some patients did not have surgical intervention but were closely monitored and their third FNAB results were reported as benign. Not including these types of patients into this study and performing evaluation retrospectively could be defined as a limitation. Prospective studies should also be conducted by evaluating both patients who have had surgery and those who have not.

In conclusion, although a significant difference was found between malignant and benign groups in terms of serum anti-thyroglobulin level and hypoechogenicity, no significant difference was observed between these groups in terms of age, sex, hormones, and other parameters of USG. Since the probability of malignancy was greater for patients who had low levels of anti-TG and hypoechoic nodules on ultrasonography, surgical treatment should strongly be recommended. It can therefore be concluded that these two parameters can be used as predictive factors for patients whose FNAB results have been nondiagnostic two or more times.

\section{REFERENCES}

1. Ghassi D, Donato A: Evaluation of the thyroid nodule. Postgrad Med J 2009; 85: 190-195.

2. Bahn RS, Castro MR: Approach to the patient with nontoxic multinodular goiter. J Clin Endocrinol Metab 2011; 96: 12021212.

3. Mijovic T, Rochon L, Gologan O, et al: Fine-needle aspiration biopsies in the management of indeterminate follicular and Hürthle cell thyroid lesions. Otolaryngol Head Neck Surg 2009; 140: 715-719.

4. Cibas ES, Ali SZ; NCl Thyroid FNA State of the Science Conference. The Bethesda System For Reporting Thyroid Cytopathology. Am J Clin Pathol. 2009 Nov;132 (5): 658-65.

5. Cooper DS, Doherty GM, Haugen BR, et al. American Thyroid Association (ATA) guidelines taskforce on thyroid nodules and differentiated thyroid cancer, revised american thyroid association management guidelines for patients with thyroid nodules and differentiated thyroid cancer. Thyroid 2009;19:1167-214.

6. Gharib H, Papini E, Paschke R et al. American association of clinical endocrinologists, associazione medici endocrinologi, and european thyroid association medical guidelines for clinical practice for the diagnosis and management of thyroid nodules. Endocr Pract 2010;16:468-75.

7. Cappelli C, Castellano M, Pirola I et all. The predictive value of ultrasound findings in the management of thyroid nodules. QJM 2007; 100:29-35.

8. Frates MC, Benson CB, Doubilet PM, et all. Prevalence and distribution ofcarcinoma in patients with solitary and multiple thyroid nodules on sonography. J Clin Endocrinol Metab 2006:91: 3411-17. 
9. Cibas ES and Ali SZ. The Bethesda System for Reporting Thyroid Cytopathology. Thyroid 2009:19:11

10. Silverman JF, West RL, Larkin EW et al. The role of fine-needle aspiration biopsy in the rapid diagnosis and management of thyroid neoplasms. Cancer 1986;57:1164-70.

11. Ashcraft MW, Van Herle AJ. Management of thyroid nodules. II: scanning techniques, thyroid suppressive therapy and fineneedle aspiration. Head Neck Surg 1981;3:297-322.

12. Alexander EK, Heering JP, Benson CB, et al. Assessment of nondiagnostic ultrasound-guided fine needle aspirations of thyroid nodules. J Clin Endocrinol Metab 2002;87:4924-7.

13. Alexander EK, Heering JP, et al. Assessment of nondiagnostic ultrasound-guided fine needle aspirations of thyroid nodules. J Clin Endocrinol Metab 2002;87:4924-7.
14. Delos SantosET, Keyhani-Rofagha S, Cunningham JJ, Mazzaferri EL. Cystic thyroid nodules. The dilemma of malignant lesions. Arch Intern Med 1990:150:1422-1427.

15. Moon HE, Kwak JY, Choi YS, et al. How to manage thyroid nodules with two consecutive non-diagnostic results on ultrasonography-guided fine needle aspiration. World J Surg 2012:36:586-592.

16. Rosario PW, Penna GC, Calsolari MR. Predictive factors of malignancy in thyroid nodules with repeatedly nondiagnostic cytology (bethesda category I): value of ultrasonography. Horm Metab Res. 2014;46(4).294-8. 